\title{
Effect of aerobic exercise in the treatment of myofascial pain: a systematic review
}

\author{
Sara Ahmed', Shereen Khattab², Chris Haddad ${ }^{3}$, Jessica Babineau', Andrea Furlan', Dinesh Kumbhare ${ }^{1, *}$ \\ 'Department of Medicine, University of Toronto, Toronto, ON, Canada \\ ${ }^{2}$ Department of Psychology, McMaster University, Hamilton, ON, Canada \\ ${ }^{3}$ Department of Physical Education \& Kinesiology, University of Toronto, Toronto, ON, Canada \\ ${ }^{4}$ Department of Library \& Information Services, University Health Network - Toronto Rehabilitation Institute, Toronto, ON, Canada
}

Myofascial pain is prevalent chronic pain disorder that is comorbid with many conditions. Strengthening and stretching exercises are capable of inducing hypoalgesic effects in people with myofascial pain syndrome. The goal of this systematic review was to summarize the effects of aerobic exercise on the management of myofascial pain. A comprehensive database search was conducted. A total of 1,331 articles were identified for review. One article was eligible for inclusion after full screening. Risk of bias was assessed using the Downs and Black quality assessment questionnaire (1998). Mean difference scores were calculated for continuous variables. The identified study had a low risk of bias. It was a randomized controlled trial assessing the influence of an 8-week water aerobic exercise program on myofascial pain in breast cancer patients. Visual analogue scale pain intensity ratings and the number of myofascial trigger points present in the neck and shoulder/axillary area of participants significantly decreased in the exercise group compared to those in the control group. Pain pressure thresholds significantly increased in muscle areas of the cervical spine in the water exercise group. Aerobic exercise presents a potential treatment modality for managing myofascial pain. Additional research is necessary.

Keywords: Myofascial pain, Exercise therapy, Myofascial pain syndrome, Physical education and training

\section{INTRODUCTION}

Myofascial pain syndrome (MPS) is a prevalent chronic pain disorder affecting approximately $85 \%$ of patients in pain clinics and $9 \%$ of the patient population in internal medicine clinics (Gerwin, 2001). At some time in their lives, $85 \%$ of the general population presents with myofascial pain (Simons, 1996). Myofascial pain is characterized by myofascial trigger points (MTrPs), which are localized hyperirritable taut bands of skeletal muscle (Lavelle et al., 2007). Hypotheses in the literature suggest that trigger points are induced by central and peripheral sensitization states as well as systemic inflammation (Fernández-de-las-Peñas and Dommerholt, 2014). Central sensitization is also thought to induce MTrP formation due to neuroplasticity within the dorsal horn that is associated with chronic peripheral nociceptive input
(Srbely et al., 2010).

Currently, the most effective methods for managing myofascial pain aim to treat MTrPs, as they are the primary triggers of generalized and referred pain in myofascial pain disorders. Common interventions include a combination of stretching and strengthening exercises, dry needling, and injections of local anaesthetics and/or corticosteroids into MTrPs, which work to overcome the energy crisis within a trigger point (Gerwin, 2014). MTrPs receive poor oxygen and energy supplies from the blood due to their contracted state. Low oxygen and energy availability contributes further to the maintenance of the contracted state within the MTrP, as adenosine triphosphate and oxygen are required to relax a muscle. The goal of myofascial pain interventions is to increase blood flow to the trigger point, which diffuses pro-inflammatory markers and brings metabolic resources to the trigger point. This
${ }^{*}$ Corresponding author: Dinesh Kumbhare (iD https://orcid.org/0000-0003-3889-7557 Department of Medicine, Division of Physical Medicine and Rehabilitation, Toronto Rehabilitation Institute, 550 University Ave, Suite 7-131, Toronto, ON M5G 2A2, Canada

Tel: +1-416-597-3422, Fax: +1-416-597-7074, E-mail: dinesh.kumbhare@uhn.ca Received: May 9, 2018 / Accepted: June 11, 2018
This is an Open Access article distributed under the terms of the Creative Commons Attribution Non-Commercial License (http://creativecommons.org/licenses/by-nc/4.0/) which permits unrestricted non-commercial use, distribution, and reproduction in any medium, provided the original work is properly cited. 
attenuates pain, reduces inflammation, and can reduce or resolve the trigger point (Ortega et al., 2009; Shah and Gilliams, 2008). Aerobic exercise achieves this effect by increasing blood pressure, flow and blood oxygen saturation allowing for more blood and metabolic substrates to enter the MTrP.

Aerobic exercise also provides many benefits against central sensitization. It is capable of inducing hypoalgesia by increasing pain pressure thresholds in muscle tissue (Drury et al., 2004; Koltyn et al., 2014; Ristic and Ellrich, 2015; Vaegter et al., 2014). In patients with fibromyalgia, aerobic exercise has been shown to reduce circulating levels of pro inflammatory markers such as interleukin (IL)-6 and IL-8 to normal levels and can also reduce substance $P$, which strengthening exercise is not capable of inducing (Mork et al., 2010; Wang et al., 2008). Aerobic exercise also promotes the production of anti-inflammatory cytokines such as IL-10 and decreases in insulin like growth factor 1 (Haydar et al., 2000; Irwin et al., 2009; Ortega et al., 2009). It potentiates the release of endogenous opioids, catecholamines, endocannabinoids, and endomorphins, which are all substances capable of inducing hypoalgesia (Dishman and O'Connor, 2009; McMurray et al., 1987). Therefore, aerobic exercise may modulate central sensitization mechanisms, increase pain thresholds, and reduce the formation of MTrPs. Aerobic exercise provides a potential noninvasive and cost effective alternative or complementary treatment to current management strategies.

The purpose of this systematic review was to identify evidence in the literature on the therapeutic benefit of aerobic exercise on myofascial pain. This review asks the following: Does aerobic exercise mitigate pain, number of MTrPs, and improve overall functionality in MPS? Results from this study will provide insight into the specific benefits of aerobic exercise on myofascial pain management, informing its prescriptive appropriateness.

\section{MATERIALS AND METHODS}

\section{Study types}

Randomized controlled trials (RCTs) and quasi-RCTs investigating the effects of aerobic exercises on myofascial pain were included in this review. Only English studies were included. Systematic reviews, case control, case reports, and cohort were excluded. Studies published only as abstracts were also excluded.

\section{Inclusion criteria for participants}

- Patients that present MTrPs.

- Presence of MTrPs confirmed by expert diagnosis.
- Meet criteria for MTrP detection as agreed upon in the literature (Kumbhare et al., 2017; Rivers et al., 2015).

A tender spot is found within palpation, with or without referral of pain (trigger point); recognition of symptoms by patient during palpation of tender spot; at least three of the following:

- Muscle stiffness of spasm.

- Limited range of motion of an associated joint.

- Pain worsens with stress.

- Palpation of taut band and/or nodule associated with a tender spot.

\section{Exclusion criteria for participants}

- Patients with inflammatory arthropathies and osteoarthritis.

- Patients chronic widespread pain.

- Patients with radiculopathy or other neurological disorders.

- Paediatric populations.

\section{Types of interventions}

- Aerobic exercise of any intensity (i.e., swimming, walking, running).

- Minimum duration of 4 weeks for intervention (an exercise intervention of less than 4 weeks is unlikely to induce therapeutic benefits (Stroth et al., 2009).

\section{Comparison group}

- Control group: no exercise.

- Alternative intervention group: treatments that do not involve exercise (e.g., dry needling, analgesics, cognitive behavioural therapy).

\section{Outcome measures}

- Pain intensity measures (e.g., visual analogue scale, VAS).

- Pain pressure thresholds using algometry.

- Use of analgesics.

- Number of trigger points determined by palpation: active and latent trigger points were combined because the pathophysiology underlying their formation is not fully understood (Gerwin, 2014).

. Functionality measures (e.g., Roland Morris Disability Questionnaire, Neck Disability Index).

- Range of motion.

\section{Search Methods for the identification of studies}

A comprehensive database search was conducted by an Information Specialist (JB) in MEDLINE (including MEDLINE ePub 
Ahead of Print, In-Process, and Other Non-Indexed Citations), Embase, the Cochrane CENTRAL Register of Controlled Trials, and Web of Science. Searches in each database were conducted from inception of the database to November 2016. The search strategy included a variety of subject headings (e.g., MeSH) and text words to describe "myofascial pain syndrome" and "exercise." Searches were limited to English language papers, and to a human population when possible. The full MEDLINE search can be reviewed in Appendix. Duplicates were removed using the citation software Endnote.

\section{Data collection}

Two reviewers independently assessed the resultant articles from the systematic search. The strategy used during the initial screening was to obtain titles and abstracts relevant to the review. Studies that did not pass the inclusion criteria were discarded and those that fulfilled the criteria remained for a full text screening. A third reviewer $\mathrm{CH}$ resolved conflicts. Once conflicts were resolved, reviewers SA and SK screened the full text articles and determined those to be included in the review. The same reviewers extracted data independently using standard data extraction forms. Where more than one publication of one trial existed, only the publication with the most complete data was included.

\section{Risk of bias}

Reviewers assessed the risk of bias within studies independently, without blinding to authorship or journal. The Downs and Black (1998) checklist for methodological quality in randomized and non-randomized studies of healthcare interventions was used to assess the following measures: selection, performance, measurement, validity, attrition, reporting of data, and statistical power.

\section{Statistical analysis}

The effect measures chosen were risk ratios for dichotomous outcomes and mean differences (MD) or standardized mean differences (SMD) for continuous data. Uncertainty was expressed using 95\% confidence intervals. Cohen criteria was used to determine the effect size of SMDs, where a SMD between 0.2 to 0.5 is small, and a SMD between 0.5 to 0.8 is moderate, and a SMD above 0.8 is large. SMDs below 0.2 were considered unsubstantial. Pooled data would be synthesized using a fixed-effects model for certain dichotomous data and a random-effects model for all continuous data. Whenever possible, we would compare fixed-effects and random-effects if there was data.

The chi-square test was used to assess heterogeneity with an al- pha of 0.05 . The $\mathrm{I}^{2}$ test for heterogeneity was used when applicable; with $<25 \%$ representing low heterogeneity, $25 \%$ to $50 \%$ representing moderate heterogeneity, and over $50 \%$ representing high heterogeneity. Clinical and methodological variability were assessed to determine sources of heterogeneity. Clinical variables may include age, duration of pain, and sex of patients. Methodological variables could include duration of exercise intervention, type of aerobic exercise, randomization, and blinding of assessors.

\section{RESULTS}

There were 1,331 articles detected by our systematic search after the removal of duplicates. Twenty-three articles were eligible for a full screen after the assessment of titles and abstracts using the inclusion and exclusion criteria. Only one study was eligible for inclusion from these articles, the remaining 22 articles did not utilize an aerobic exercise intervention but rather strengthening and stretching exercises, or a combination of both. Fig. 1 outlines the Preferred Reporting Items for Systematic Reviews and Meta-Analyses flow diagram of the search.

The study done by Cantarero-Villanueva et al. (2012) was the only study that met our inclusion/exclusion criteria. This RCT assessed the impact of aerobic swimming exercise supplemented with strengthening and stretching exercises in breast cancer patients with MTrPs and cervical and shoulder-axillary pain. Patients who have undergone a mastectomy, were not on any treatment except hormone therapy, and had myofascial pain were included. Seventy patients were enrolled in the study after a $90 \%$ powered analysis. Four subjects were excluded prior to the intervention and the reason was not provided. Sixty-six patients were equally randomized using computer-generated numbers into two groups, a water exercise intervention or a control group. One participant did not complete the intervention and the reason was not provided. The intervention group engaged in a water exercise program three times a week for eight consecutive weeks. Each session was held in a warm pool for one hour. The first 10 min was warm up which consisted of slow aerobic movements and stretching. The next $35 \mathrm{~min}$ consisted of low intensity endurance and core stability training. The final $15 \mathrm{~min}$ were a cool down period along with neck and shoulder stretching and relaxation exercises. Patient outcomes were assessed at baseline and after the 8-week intervention. The study outcomes were (a) VAS scores for neck and shoulder-axillary pain, (b) pressure pain threshold in the affected and unaffected sides of the zygapophyseal joints, deltoid, second metacarpal, and tibialis anterior muscles, (c) number and 


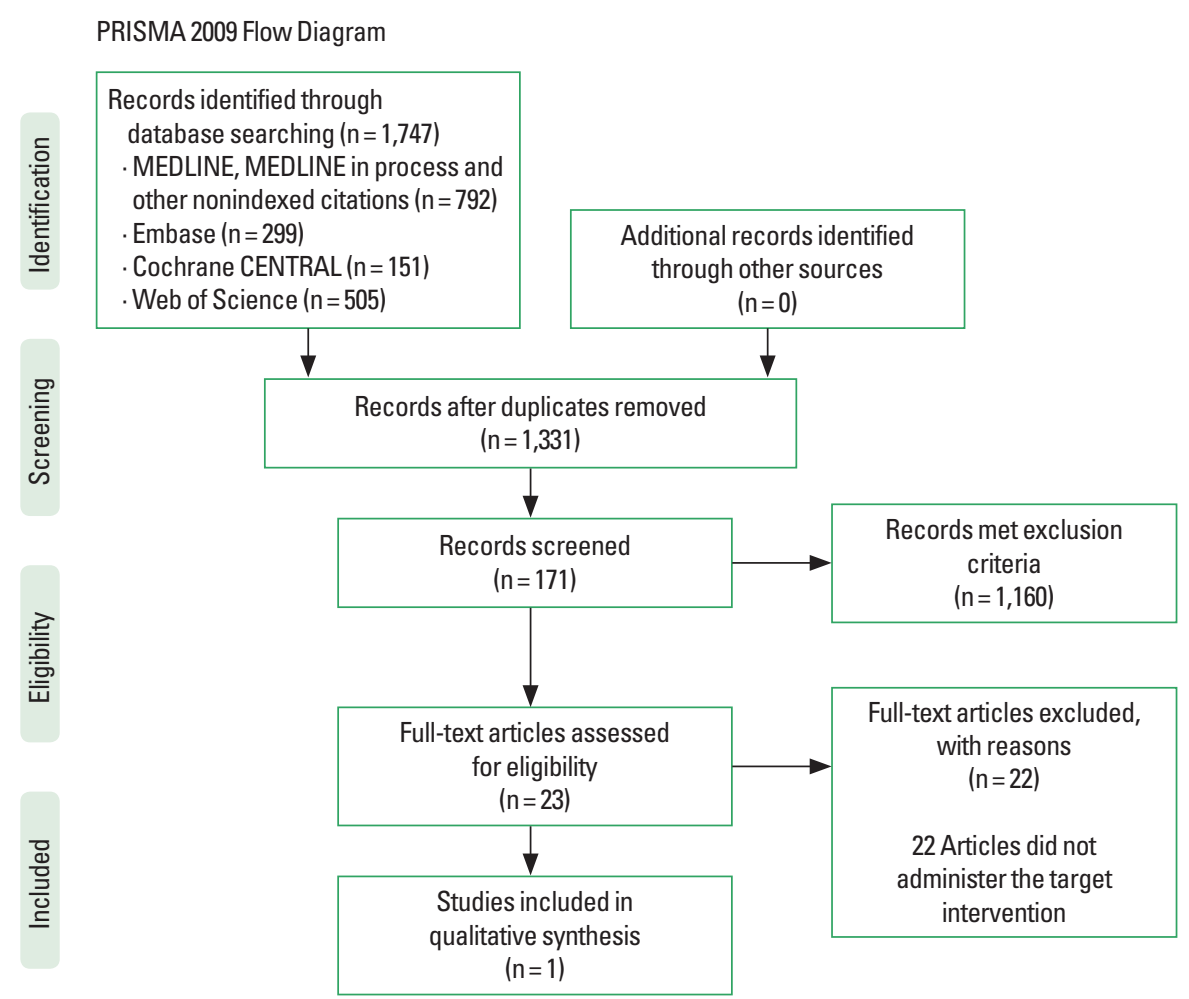

Fig. 1. Preferred Reporting Items for Systematic Reviews and Meta-Analyses (PRISMA) flow diagram. Adapted from Moher et al. (2009).

location of active and latent trigger points in the affected and unaffected sides of the upper trapezius, levator scapulae, scalene, pectoralis major, and infraspinatus muscles.

\section{Risk of bias assessment}

According to Downs and Black's (1998) checklist for methodological quality in randomized and non-randomized studies of healthcare interventions the study of Cantarero-Villanueva et al. (2012) study was determined to have low risk of bias, except for some risk of performance bias. This was due to the lack of clarity to whether investigators where blinded from the intervention assignment. The authors clearly reported their aim, outcome measures and methods, patient characteristics, intervention, confounders, results, and attrition. Accordingly, the external validity of the study was adequate. The study contained consecutive patients attending the Breast Oncology Unit of the Hospital Virgen de las Nieves and Hospital Clínico San Cecilio, Granada (Spain) from June 2010 to September 2011. Randomization procedures were computer generated. Only assessors were blinded to the intervention since patients were receiving an overt treatment. $\mathrm{Pa}$ tient compliance was adequately measured. Patients in the treatment group completed $85 \%$ of the exercise sessions. Control par- ticipants were asked about their physical activity during the study period to ensure their physical activity did not equal or exceed that of the intervention group. The sample size was adequate since a minimum of fifteen participants were required in each group to achieve a power of $90 \%$, however 32 and 33 individuals were in the intervention and control group, respectively. Thus the study was sufficiently powered.

\section{Effects of interventions}

This RCT compared pain (by VAS), pain pressure thresholds, and number of MTrPs in the cervical and shoulder area of breast cancer survivors with neck and shoulder-axillary pain before and after a water exercise intervention. The authors reported significant changes in all three outcomes after the exercise intervention. Tables 1 and 2 summarize the results of the study.

There were significant differences in pain intensity scores after the water exercise intervention in the neck and shoulder/axillary area between participants in the water exercise group and the control group. There was variability in the observed pain pressure thresholds between participants in the water exercise group and the control group. The authors reported significant pain pressure threshold differences between the water exercise group and the 
Table 1. Mean difference scores for visual analogue scale (VAS) scores and pain pressure thresholds in the water exercise and control groups before and after treatment

\begin{tabular}{|c|c|c|c|}
\hline Variable & Preintervention & Postintervention & $\begin{array}{c}\text { Mean difference } \\
(95 \% \mathrm{CI})\end{array}$ \\
\hline \multicolumn{4}{|l|}{ Change in VAS scores } \\
\hline \multicolumn{4}{|l|}{ Neck pain } \\
\hline Water exercise group & $40 \pm 31$ & $12 \pm 15$ & $-30(-25$ to -35$)$ \\
\hline Control group & $39 \pm 21$ & $42 \pm 23$ & \\
\hline \multicolumn{4}{|l|}{ Shoulder/axillary pain } \\
\hline Water exercise group & $27 \pm 33$ & $12 \pm 13$ & -31 (-25 to -37$)$ \\
\hline Control group & $38 \pm 35$ & $43 \pm 33$ & \\
\hline \multicolumn{4}{|c|}{ Change in pain pressure thresholds } \\
\hline \multicolumn{4}{|l|}{ C5-6 zygapophyseal joint } \\
\hline $\begin{array}{l}\text { Water exercise group } \\
\text { affected }\end{array}$ & $156.0 \pm 65.8$ & $174.0 \pm 63.5$ & 20.3 (34.1 to 6.5) \\
\hline Control group affected & $163.4 \pm 62.7$ & $153.7 \pm 49.7$ & \\
\hline \multicolumn{4}{|l|}{ C5-6 zygapophyseal joint } \\
\hline $\begin{array}{l}\text { Water exercise group } \\
\text { unaffected }\end{array}$ & $167.4 \pm 66.3$ & $167.3 \pm 62.4$ & 3.6 (17.8 to -10.6$)$ \\
\hline $\begin{array}{r}\text { Control group } \\
\text { unaffected }\end{array}$ & $181.9 \pm 61.8$ & $163.7 \pm 54.1$ & \\
\hline \multicolumn{4}{|l|}{ Affected deltoid muscle } \\
\hline Water exercise group & $181.4 \pm 76.8$ & $206.9 \pm 66.8$ & -6.7 (9.9 to -23.3$)$ \\
\hline Control group & $194.6 \pm 111.4$ & $213.6 \pm 69.3$ & \\
\hline \multicolumn{4}{|l|}{ Unaffected deltoid muscle } \\
\hline Water exercise group & $191.6 \pm 83.3$ & $212.6 \pm 61.1$ & $-10.5(4.3$ to -25.3$)$ \\
\hline Control group & $223.4 \pm 77.7$ & $223.1 \pm 60.4$ & \\
\hline \multicolumn{4}{|c|}{ Affected second metacarpal } \\
\hline Water exercise group & $210.4 \pm 65.9$ & $243.8 \pm 83.8$ & 22.1 (40.5 to 3.7) \\
\hline Control group & $234.4 \pm 81.1$ & $221.7 \pm 66.9$ & \\
\hline \multicolumn{4}{|c|}{ Unaffected second metacarpal } \\
\hline Water exercise group & $242.3 \pm 66.3$ & $255.2 \pm 72.6$ & 6.4 (22.3 to -9.5$)$ \\
\hline Control group & $233.1 \pm 80.9$ & $248.8 \pm 57.7$ & \\
\hline \multicolumn{4}{|c|}{ Affected tibialis anterior muscle } \\
\hline Water exercise group & $261.2 \pm 108.6$ & $259.4 \pm 82.1$ & $-52.8(-32.0$ to -73.6$)$ \\
\hline Control group & $293.3 \pm 83.8$ & $312.2 \pm 88.5$ & \\
\hline \multicolumn{4}{|c|}{ Unaffected tibialis anterior muscle } \\
\hline Water exercise group & $275.4 \pm 112.5$ & $268.5 \pm 101.7$ & $-48.6(-26.9$ to -70.3$)$ \\
\hline Control group & $302.9 \pm 92.1$ & $317.1 \pm 75.4$ & \\
\hline
\end{tabular}

Values are presented as mean \pm standard deviation unless otherwise indicated. $\mathrm{Cl}$, confidence interval.

control group after the intervention zygapophyseal joint and no other areas.

The authors reported significantly less active MTrPs in the water exercise group after the intervention in all affected muscle areas but the sternocleidomastoid muscle. The experimental group also had a greater reduction in the number of MTrPs relative to the control group. In all unaffected muscles areas, there was sig-
Table 2. Number of myofascial trigger points in the water exercise and control groups before and after the exercise treatment

\begin{tabular}{|c|c|c|}
\hline Variable & Preintervention & Postintervention \\
\hline \multicolumn{3}{|c|}{ Affected upper trapezius muscle } \\
\hline Water exercise group & 18 & 5 \\
\hline Control group & 18 & 19 \\
\hline \multicolumn{3}{|c|}{ Unaffected upper trapezius muscle } \\
\hline Water exercise group & 19 & 5 \\
\hline Control group & 14 & 14 \\
\hline \multicolumn{3}{|c|}{ Affected sternocleidomastoid muscle } \\
\hline Water exercise group & 9 & 1 \\
\hline Control group & 10 & 16 \\
\hline \multicolumn{3}{|c|}{ Unaffected sternocleidomastoid muscle } \\
\hline Water exercise group & 9 & 3 \\
\hline Control group & 6 & 11 \\
\hline \multicolumn{3}{|c|}{ Affected infraspinatus muscle } \\
\hline Water exercise group & 10 & 3 \\
\hline Control group & 16 & 16 \\
\hline \multicolumn{3}{|c|}{ Unaffected infraspinatus muscle } \\
\hline Water exercise group & 10 & 2 \\
\hline Control group & 12 & 13 \\
\hline \multicolumn{3}{|l|}{ Affected scalene muscle } \\
\hline Water exercise group & 24 & 6 \\
\hline Control group & 18 & 17 \\
\hline \multicolumn{3}{|l|}{ Unaffected scalene muscle } \\
\hline Water exercise group & 14 & 4 \\
\hline Control group & 6 & 2 \\
\hline \multicolumn{3}{|c|}{ Affected pectoralis major muscle } \\
\hline Water exercise group & 25 & 10 \\
\hline Control group & 21 & 27 \\
\hline \multicolumn{3}{|c|}{ Unaffected pectoralis major muscle } \\
\hline Water exercise group & 4 & 1 \\
\hline Control group & 6 & 2 \\
\hline \multicolumn{3}{|c|}{ Affected infraspinatus muscle } \\
\hline Water exercise group & 12 & 2 \\
\hline Control group & 12 & 18 \\
\hline \multicolumn{3}{|c|}{ Unaffected infraspinatus muscle } \\
\hline Water exercise group & 8 & 0 \\
\hline Control group & 5 & 11 \\
\hline
\end{tabular}

nificant decrease in trigger points after the intervention. Cantarero-Villanueva et al. (2012) reported only mean number of MTrPs and no variability measures, thus MD scores could not be calculated.

\section{DISCUSSION}

One RCT met the inclusion criteria for this study. The study by Cantarero-Villanueva et al. (2012) was adequately powered, 
well controlled, accounted for potential confounders, and had low attrition providing reliable results. The results suggest that water based aerobic exercise is an effective treatment for myofascial pain. Patients had significantly lower neck and shoulder-axillary pain after the intervention as well as higher pain pressure thresholds over the cervical spine. The number of MTrPs was significantly reduced among participants in the aerobic exercise treatment group after the intervention relative to the control group. Results from this study indicate that aerobic exercise may be modulating central sensitization mechanisms thereby reducing or preventing the formation of MTrPs.

These findings present effects similar to those found in the literature on strengthening exercise and myofascial pain. Isometric strengthening exercises have been shown to reduce VAS pain scores and disability indices in MPS (Acar and Yilmaz, 2012; Buttagat et al., 2016; Cho et al., 2012; Trampas et al., 2010). The benefits received from these interventions suggest that exercise may be increasing blood supply and metabolic resources to MTrPs possibly through the mechanical displacement of muscle fibres. Strengthening and aerobic exercises probably results in local increases in blood pressure with improvement in blood flow to resistant areas. This may improve the increased resistance of the vascular bed that characterizes the MTrP (Ballyns et al., 2011; Sikdar et al., 2009; Turo et al., 2013).

In this study, pain pressure thresholds increased only in the cervical spine muscles of the water exercise group. This muscle group is utilized extensively during water-based activities and therefore may have been strengthened to a greater extent than other muscle groups. This is potentially the explanation for the increased pain threshold observed in the cervical area. Several studies investigating the effects of strengthening exercises on muscle groups associated with MPS have also reported similar results (Buttagat et al., 2016; Trampas et al., 2010). Buttagat et al. (2016) found significant improvements in pain intensity and pain pressure thresholds after patients with myofascial pain in the scapular area were administered scapular stabilizing exercises. Trampas et al. (2010) also found increases in pain pressure thresholds after strengthening exercise but no reduction in the number of trigger points present. These studies suggest that aerobic exercise and strengthening exercise influence different but complementary mechanisms to alleviate myofascial pain. Aerobic exercise may reduce the presence of generalized pain and number of MTrPs, whereas strengthening exercises may provide additional support to affected muscle groups and increase pain tolerance. More studies are needed to assess outcomes and mechanisms influenced by aerobic exercise in people with myofascial pain.

This systematic review provides the first review assessing the benefits of aerobic exercise on MPS. The systematic search for this review was extensive, encompassing a wide range of exercise terms and four scientific and medical databases were used to locate articles studying the effect of this intervention. Only one study (Cantarero-Villanueva et al., 2012) met our inclusion and exclusion criteria. It had a low risk of bias, presenting strong evidence for the potential utility of aerobic exercise as a non-pharmacological intervention for MPS. Several outcome measures were assessed in this study, which provided insight into the specific effects aerobic exercise may have on myofascial pain, namely the pain intensity (VAS), pain pressure thresholds, and the number of MTrPs. There is a need to identify feasible and economical treatments that improve the well-being and quality of life in people living with MPS. Cantarero-Villanueva et al. (2012) findings provide evidence that aerobic exercise is an effective and practical intervention.

Several limitations are present when conducting this review. Utilizing palpation to detect MTrPs has poor inter-rater reliability, and thus may have influenced the MTrP count reported by Cantarero-Villanueva et al. (2012). Their study used one unblended assessor to perform the MTrP count. There is the risk of expectation bias with this approach. However, this could have mitigated the poor interrater reliability associated with palpation if the assessor was accurate at detecting MTrPs. However, if the accuracy was low or variable the reported outcomes may have been biased. Another limitation was that the studies included in this review were English language publications only, which may have produced further bias. A final limitation was that only one article was identified limiting the conclusions on the effects of aerobic exercise on myofascial pain.

Despite these limitations, the risk of bias in this study was low, which lends to the strength of its conclusions. No intervention related adverse effects were reported in this study. Therefore, clinicians may consider recommending aerobic exercise to help manage patients' myofascial pain symptoms. Based on the available evidence, aerobic exercise in combination with strengthening and stretching exercises would be effective at managing myofascial pain due to their complementary effects on pain mitigation, reduction of trigger point formation, and increasing pain tolerance. The appropriate exercise prescription should be personalized and observe any specific patient related contraindications. Further research is necessary to elucidate the effects of aerobic exercise alone or in combination with other types of exercise or treatment methods such as dry needling on MPS. Furthermore, future research 
should investigate the benefits of aerobic exercise on the pathophysiological mechanisms of myofascial pain, such as assessing inflammatory or stress response changes. This will better elucidate the role of aerobic exercise in managing MPS and potentially other chronic pain disorders. Previous research has demonstrated that women are more responsive to exercise induced hypoalgesia after aerobic exercise relative to men (Vaegter et al., 2014). Investigating gender differences in hypoalgesia responses after aerobic exercise between males and females living with myofascial pain should also be considered.

\section{CONFLICT OF INTEREST}

No potential conflict of interest relevant to this article was reported.

\section{REFERENCES}

Acar B, Yllmaz OT. Effects of different physiotherapy applications on pain and mobility of connective tissue in patients with myofascial pain syndrome. J Back Musculoskelet Rehabil 2012;25:261-267.

Ballyns JJ, Shah JP, Hammond J, Gebreab T, Gerber LH, Sikdar S. Objective sonographic measures for characterizing myofascial trigger points associated with cervical pain. J Ultrasound Med 2011;30:1331-1340.

Buttagat V, Taepa N, Suwannived N, Rattanachan N. Effects of scapular stabilization exercise on pain related parameters in patients with scapulocostal syndrome: a randomized controlled trial. J Bodyw Mov Ther 2016;20:115-122.

Cantarero-Villanueva I, Fernández-Lao C, Fernández-de-Las-Peñas C, López-Barajas IB, Del-Moral-Ávila R, de la-Llave-Rincón AI, ArroyoMorales M. Effectiveness of water physical therapy on pain, pressure pain sensitivity, and myofascial trigger points in breast cancer survivors: a randomized, controlled clinical trial. Pain Med 2012;13:15091519.

Cho YS, Park SJ, Jang SH, Choi YC, Lee JH, Kim JS. Effects of the combined treatment of extracorporeal shock wave therapy (ESWT) and stabilization exercises on pain and functions of patients with myofascial pain syndrome. J Phys Ther Sci 2012;24:1319-1323.

Dishman RK, O'Connor PJ. Lessons in exercise neurobiology: the case of endorphins. Ment Health Phys Act 2009;2:4-9.

Downs SH, Black N. The feasibility of creating a checklist for the assessment of the methodological quality both of randomised and non-randomised studies of health care interventions. J Epidemiol Community Health 1998;52:377-384.

Drury DG, Stuempfle KJ, Shannon RJ, Miller JL. An investigation of exer- cise-induced hypoalgesia after isometric and cardiovascular exercise. J Exerc Physiol 2004;7:1-5.

Fernández-de-las-Peñas C, Dommerholt J. Myofascial trigger points: peripheral or central phenomenon? Curr Rheumatol Rep 2014;16:395.

Gerwin RD. Classification, epidemiology, and natural history of myofascial pain syndrome. Curr Pain Headache Rep 2001;5:412-420.

Gerwin RD. Diagnosis of myofascial pain syndrome. Phys Med Rehabil Clin N Am 2014;25:341-355.

Haydar ZR, Blackman MR, Tobin JD, Wright JG, Fleg JL. The relationship between aerobic exercise capacity and circulating IGF-1 levels in healthy men and women. J Am Geriatr Soc 2000;48:139-145.

Irwin ML, Varma K, Alvarez-Reeves M, Cadmus L, Wiley A, Chung GG, Dipietro L, Mayne ST, Yu H. Randomized controlled trial of aerobic exercise on insulin and insulin-like growth factors in breast cancer survivors: the Yale Exercise and Survivorship study. Cancer Epidemiol Biomarkers Prev 2009;18:306-313.

Koltyn KF, Brellenthin AG, Cook DB, Sehgal N, Hillard C. Mechanisms of exercise-induced hypoalgesia. J Pain 2014;15:1294-1304.

Kumbhare D, Shaw S, Grosman-Rimon L, Noseworthy MD. Quantitative ultrasound assessment of myofascial pain syndrome affecting the trapezius: a reliability study. J Ultrasound Med 2017;36:2559-2568.

Lavelle ED, Lavelle W, Smith HS. Myofascial trigger points. Anesthesiol Clin 2007;25:841-851.

McMurray RG, Forsythe WA, Mar MH, Hardy CJ. Exercise intensity-related responses of beta-endorphin and catecholamines. Med Sci Sports Exerc 1987;19:570-574.

Moher D, Liberati A, Tetzlaff J, Altman DG; PRISMA Group. Preferred reporting items for systematic reviews and meta-analyses: the PRISMA statement. PLoS Med 2009;6:e1000097.

Mork PJ, Vasseljen O, Nilsen TI. Association between physical exercise, body mass index, and risk of fibromyalgia: longitudinal data from the Norwegian Nord-Trøndelag Health Study. Arthritis Care Res (Hoboken) 2010;62:611-617.

Ortega E, García JJ, Bote ME, Martín-Cordero L, Escalante Y, Saavedra JM, Northoff H, Giraldo E. Exercise in fibromyalgia and related inflammatory disorders: known effects and unknown chances. Exerc Immunol Rev 2009;15:42-65.

Ristic D, Ellrich J. Exercise-induced hypoalgesia differentially affects deep pain, cutaneous pain and innocuous somatosensation. Acta Physiol 2015;213:63-64.

Rivers WE, Garrigues D, Graciosa J, Harden RN. Signs and symptoms of myofascial pain: an international survey of pain management providers and proposed preliminary set of diagnostic criteria. Pain Med 2015; 16:1794-1805.

Shah JP, Gilliams EA. Uncovering the biochemical milieu of myofascial 
trigger points using in vivo microdialysis: an application of muscle pain concepts to myofascial pain syndrome. J Bodyw Mov Ther 2008; 12:371-384.

Sikdar S, Shah JP, Gebreab T, Yen RH, Gilliams E, Danoff J, Gerber LH. Novel applications of ultrasound technology to visualize and characterize myofascial trigger points and surrounding soft tissue. Arch Phys Med Rehabil 2009;90:1829-1838.

Simons DG. Clinical and etiological update of myofascial pain from trigger points. J Musc Pain 1996;4:93-122.

Srbely JZ, Dickey JP, Bent LR, Lee D, Lowerison M. Capsaicin-induced central sensitization evokes segmental increases in trigger point sensitivity in humans. J Pain 2010;11:636-643.

Stroth S, Hille K, Spitzer M, Reinhardt R. Aerobic endurance exercise benefits memory and affect in young adults. Neuropsychol Rehabil 2009; 19:223-243.
Trampas A, Kitsios A, Sykaras E, Symeonidis S, Lazarou L. Clinical massage and modified Proprioceptive Neuromuscular Facilitation stretching in males with latent myofascial trigger points. Phys Ther Sport 2010;11:91-98.

Turo D, Otto P, Shah JP, Heimur J, Gebreab T, Zaazhoa M, Armstrong K, Gerber LH, Sikdar S. Ultrasonic characterization of the upper trapezius muscle in patients with chronic neck pain. Ultrason Imaging 2013; 35:173-187.

Vaegter HB, Handberg G, Graven-Nielsen T. Similarities between exerciseinduced hypoalgesia and conditioned pain modulation in humans. Pain 2014;155:158-167.

Wang H, Moser M, Schiltenwolf M, Buchner M. Circulating cytokine levels compared to pain in patients with fibromyalgia: a prospective longitudinal study over 6 months. J Rheumatol 2008;35:1366-1370. 


\section{Appendix 1. MEDLINE search strategy}

Database: Epub Ahead of Print, In-Process \& Other Non-Indexed Citations, Ovid MEDLINE(R) Daily and Ovid MEDLINE(R) $<1946$ to Present $>$

Search strategy:

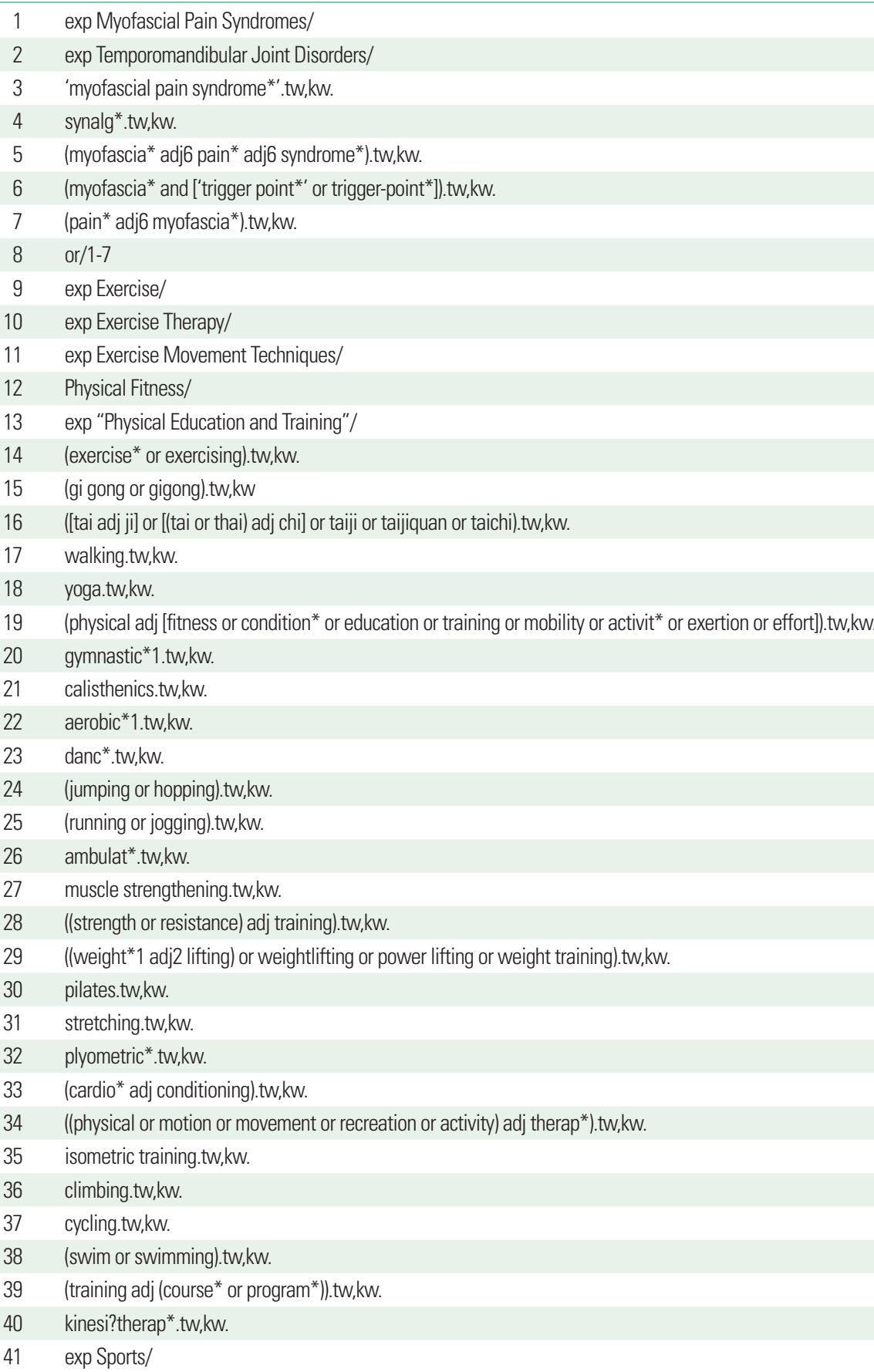

